\title{
Klassische Formulierungen weiterhin gefragt
}

? Können neue Vehikelsysteme wie DMS-Cremes oder nanodisperse Träger die etablierten Wasser-in-Öl- und Öl-in-WasserZubereitungen als Grundlage von Dermatokosmetika ablösen? Daniels: Ich sehe in den neuen Systemen durchaus Vorteile, aber sie werden die klassischen Formulierungen nicht komplett ersetzen. DMS ist eine Alternative zu den mit klassischen Emulgatoren stabilisierten Zubereitungen. Physikochemisch ist DMS ein Öl-in-Wasser-System, die Wirkung an der Haut geht in Richtung Wasser-in-Öl-System. Der Marktanteil an DMS liegt grob geschätzt derzeit aber höchstens bei $20 \%$.

Bei den nanodispersen Trägersystemen ist die primäre Intention, einen Wirkstoff besser in die Haut zu transportieren, um dort eine Art Depot anzulegen. Sie werden normalerweise nicht allein als Pflegeprodukt verwendet, sondern einer geeigneten Grundformulierung zugesetzt. Werden Liposomen verwendet, haben die enthaltenen Phospholipide natürlich auch eine gewisse pflegende Wirkung.

? Wo liegen die Vorteile der neueren Grundlagenformulierungen für den Verbraucher?

Daniels: DMS-Cremes enthalten Phospholipide, die zu den Emulgatoren gehören, in Wasser aber nicht löslich sind und keine Mizellen bilden, sie sind also nicht waschaktiv. Das ist der große Unterschied zu konventionellen Öl-in-Wasser-Cremes, in denen man wasserlösliche Emulgatoren einsetzt, die Hautlipide auswaschen können. Indem man nur hautverwandte Lipide verwendet, kann man mutmaßen, dass fehlende Hautlipide durch die DMS-Mischung substituiert werden können. In Bezug auf die Barrierestärkung hat man wegen der fehlenden hydrophilen Emulgatoren von DMS-Cremes einen ähnlichen Effekt zu erwarten wie von klassischen Wasser-in-Öl-Cremes. Vorteile haben allerdings nur Verbraucher, bei denen tatsächlich eine Barrierestörung vorliegt.

Bei der Wirkkosmetik soll durch nanodisperse Trägersysteme der Effekt gesteigert werden. Man darf aber nicht vergessen, dass in $90 \%$ der Fälle mit einer effizienten Penetrationsförderung auch eine Barriereschädigung einhergeht. Eigentlich gehört dieses Prinzip eher in den Bereich der Arzneiwirkstoffe.

? Sind die neueren Formulierungen zur Basispflege bei speziellen Dermatosen besser geeignet als herkömmliche?

Daniels: In diesem Bereich können nanodisperse Trägersysteme gefragt sein, man kann sie aber nicht speziell einzelnen Dermatosen zuordnen; dies bestimmt der enthaltene Wirkstoff.

Bei einer Akne sollte die Formulierung natürlich möglichst fettarm und austrocknend sein. Wenn Wirkstoffe eingebracht werden sollen, sollte es die Formulierung schaffen, den Talgpfropf, der die Pore verschließt, zu erweichen, damit der Wirkstoff in die Läsion gelangen kann. Eine waschaktive Substanz etwa kann den Talg emulgieren und wirkt eher austrocknend.

Trockene Haut dagegen braucht Fett und Feuchtigkeit sowie wasserspeichernde Substanzen. Beim atopischen Ekzem und trockenem Hautzustand ist eine DMS-Grundlage durchaus

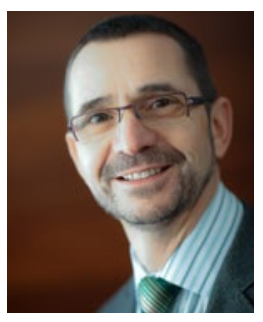

Prof. Dr. Rolf Daniels

Pharmazeutische Technologie

Eberhard Karls Universität

Tübingen

sinnvoll, aber auch eine klassische W/O-Formulierung. Kosmetische Wirkstoffe wie Harnstoff, die in die Haut transportiert werden, erhöhen zudem die Hautfeuchte.

Trockene Altershaut oder empfindliche Haut werden ähnlich wie die Haut des Atopikers gepflegt. Neuere Untersuchungen geben Hinweise darauf, dass für die Altershaut der $\mathrm{pH}$ noch etwas saurer eingestellt werden sollte, also deutlich unter $\mathrm{pH} 5$.

Bei der Psoriasis ist die rein pflegende Komponente ebenfalls ähnlich wie beim Atopiker, die einzubringenden Wirkstoffe dienen allerdings dazu, die Hautschuppen abzulösen.

\section{? Welche Folgen können auftreten, wenn falsche Grundlagen} verwendet werden?

Daniels: Eine gesunde Haut braucht eigentlich nicht sonderlich viel Pflegeprodukte. Verwendet man bei einer Haut mit normalem Wassergehalt und ausgewogener Barrierefunktion zu viel Feuchtigkeitscreme, kann es zur Überfeuchtung kommen. Bringt man reichlich Öl-in-Wasser-Emulsion mit vielen Emulgatoren auf, die die Lipidbestandteile auswaschen, kann auch die Barriere einer normalen Haut Schaden nehmen. Ein gutes Beispiel ist die periorale Dermatitis, die sogenannte Stewardessenkrankheit. In der extrem trockenen Luft wird das Gesicht sehr viel eingecremt. Als Reaktion der Haut auf das Überpflegen entsteht eine heftige Entzündung um den Mund, die man nur in den Griff bekommt, indem man mehrere Wochen lang in diesem Bereich keinerlei Pflegeprodukte anwendet.

Bei bereits erkrankter Haut geschieht dies genauso, nur dass der Hautzustand dann ohnehin schon in Richtung zu trocken oder zu feucht verschoben ist.

? Mit welchen Neuentwicklungen bei den Trägersystemen ist in naher Zukunft zu rechnen?

Daniels: Bei den klassischen Formulierungen wird man auch weiterhin nach hautverträglicheren Emulgatoren suchen. DMSSysteme versucht man noch besser an die Hautlipide anzupassen. Nanopartikuläre Trägersysteme werden zum Teil weiterentwickelt, aber auch hier ist das Wesentliche bereits geschehen. Ich sehe in absehbarer Zeit nirgendwo eine echte Sprunginnovation am Horizont.

Das Interview führte Dr. Christine Starostzik. 